\title{
AVALIAÇÃO DO VOLUME DE OXIGÊNIO ABSORVIDO POR SACHÊ ABSORVEDOR DE OXIGÊNIO EM DIFERENTES TEMPERATURAS E UMIDADES RELATIVAS
}

\author{
Evaluation of the amount of oxygen absorbed by oxygen absorbers at \\ different temperatures and relative humidities
}

Renato Souza Cruz ${ }^{1}$, Nilda de Fátima Ferreira Soares ${ }^{2}$, Robson Maia Geraldine ${ }^{3}$

\begin{abstract}
RESUMO
O uso de absorvedores de oxigênio em embalagens de produtos alimentícios acondicionados tem apresentado uma demanda crescente. Assim, o conhecimento da eficiência desses absorvedores em diferentes condições de umidade relativa e temperaturas definidas, são de fundamental importância. Portanto, foram determinadas equações para predizer o volume absorvido de oxigênio para as temperaturas de $10 \pm 2{ }^{\circ} \mathrm{C}$ e $25 \pm 2{ }^{\circ} \mathrm{C}$, dependendo da umidade relativa na faixa de $75 \%$ a $85 \%$ e da taxa de permeabilidade a oxigênio da embalagem. Para a temperatura de $25 \pm 2^{\circ} \mathrm{C}$ a equação é: $\mathrm{V}=-32,770+10,440 * \mathrm{UR}-104,385 * \ln \left(\mathrm{TPO}_{2}\right)$, com um $\mathrm{R}^{2}=$ 0,9151 . Para a temperatura de $10 \pm 2^{\circ} \mathrm{C}$ a equação é: $\mathrm{V}=107,321+6,221 * \mathrm{UR}-105,166 \ln \left(\mathrm{TPO}_{2}\right)$ com um $\mathrm{R}^{2}=0,8729$. Dessa forma, o tempo de atividade do sachê pode ser determinado pela equação $\mathrm{T}=(\mathrm{V}-\mathrm{Vi}) /\left(\mathrm{TPO}_{2} * \mathrm{~A}\right)$. Utilizando essas equações e, considerando uma embalagem de área $0,05 \mathrm{~m}^{2}$ por face, com uma permeabilidade de $8,63 \mathrm{~cm}^{3} \cdot \mathrm{m}^{-2}$. dia $\mathrm{di}^{-1}$, uma umidade relativa de $80 \%$ e o volume de oxigênio inicial dentro da embalagem de $2,5 \mathrm{~mL}$, após o envase, o tempo de atividade do sachê quando armazenado a $10 \pm 2^{\circ} \mathrm{C}$ foi de 435 dias e a $25 \pm 2^{\circ} \mathrm{C}$ de 666 dias.
\end{abstract}

Termos para indexação: Embalagem ativa, absorvedor de oxigênio, curva de absorção.

\section{ABSTRACT}

Oxygen absorbers have been presenting a growing demand for application in food packaging. Thus, it is important to know the efficiency of those absorbers in different relative humidity and temperatures. Therefore, equations were developed to predict the volume of absorbed oxygen at $10 \pm 2{ }^{\circ} \mathrm{C}$ and $25 \pm 2{ }^{\circ} \mathrm{C}$, according as the relative humidity ranging from $75 \%$ to $85 \%$ and the oxygen transmission rate through the package. At $25 \pm 2^{\circ} \mathrm{C}$ the equation was $\mathrm{V}=-32,770+10,440 * \mathrm{RH}-104,385 * \ln \left(\mathrm{O}_{2} \mathrm{TR}\right)$, with $\mathrm{R}^{2}=0,9151$. At $10 \pm 2^{\circ} \mathrm{C}, \mathrm{V}=107,321+6,221 * \mathrm{RH}-105,166 \ln \left(\mathrm{O}_{2} \mathrm{TR}\right)$ with $\mathrm{R}^{2}=0,8729$. As a consequence, activity time for the oxygen absorbers can be calculated with the following equation: $\mathrm{T}=(\mathrm{V}-\mathrm{Vi}) /\left(\ln \left(\mathrm{O}_{2} \mathrm{TR} * \mathrm{~A}\right)\right.$. Using these equations and considering a packaging area of $0,05 \mathrm{~m}^{2}$ for each face, oxygen transmission rate of $8,63 \mathrm{~cm}^{3} \cdot \mathrm{m}^{-2} \cdot \mathrm{dia}^{-1}$, relative humidity of $80 \%$ and an initial oxygen volume inside the package of $2,5 \mathrm{~mL}$, absorber activity times when stored at $10 \pm 2^{\circ} \mathrm{C}$ and $25 \pm 2^{\circ} \mathrm{C}$ were 435 and 666 days, respectively.

Index terms: Active packaging, oxygen absorbers, absorbed oxygen.

(Recebido em 22 de setembro de 2004 e aprovado em 21 de agosto de 2006)

\section{INTRODUÇÃO}

Os absorvedores de $\mathrm{O}_{2}$, comercialmente disponíveis, estão em formas de pequenos sachês, contendo agentes metálicos redutores, incluindo o óxido de ferro em pó, carbonato ferroso e platina metálica. De forma geral, a tecnologia dos absorvedores baseia-se na oxidação de pó de ferro, ácido ascórbico ou da ação de polímero fotossensível, enzimas, entre outros (FLOROS et al., 1997; LABUZA \& BREENE, 1989; ROONEY, 1995; VERMEIREN et al., 1999). Os absorvedores de constituição metálica utilizam o princípio da oxidação do ferro na presença de água (SMITH et al., 1990). Segundo McKedy
(2002) as partículas de ferro são prontamente combinadas com o $\mathrm{O}_{2}$, por isso são muito utilizadas. Porém, em ambiente seco é necessária adição de um composto absorvedor de umidade para produzir uma ação eletrolítica necessária para ativar o absorvedor de $\mathrm{O}_{2}$.

Segundo Abe \& Kondoh (1989), o mecanismo é muito complexo para ser expresso por uma fórmula simples, mas geralmente é representado como:

$$
\begin{aligned}
& \mathrm{Fe} \rightarrow \mathrm{Fe}^{+2}+2 \mathrm{e}^{-} \\
& 1 / 2 \mathrm{O}_{2}+\mathrm{H}_{2} \mathrm{O}+2 \mathrm{e}^{-} \rightarrow 2 \mathrm{OH}^{-} \\
& \mathrm{Fe}^{2}+2 \mathrm{OH}^{-} \rightarrow \mathrm{Fe}(\mathrm{OH})_{2} \\
& \mathrm{Fe}(\mathrm{OH})_{2}+1 / 4 \mathrm{O}_{2}+1 / 2 \mathrm{H}_{2} \mathrm{O} \rightarrow \mathrm{Fe}(\mathrm{OH})_{3}
\end{aligned}
$$

\footnotetext{
'Engenheiro de Alimenos, Doutor, Professor - Departamento de Tecnologia - Universidade Estadual de Feira de Santana/UEFS - Avenida Universitária, $\mathrm{s} / \mathrm{n}$ - Br 116, Km 03 - Campus Universitário - 44031-460 - Feira de Santana, BA - cruz.rs@gmail.com

${ }^{2}$ Doutora, Professora - Departamento de Tecnologia de Alimentos/DTA - Universidade Federal de Viçosa/UFV - Avenida PH Rolfs, s/n - Campus Universitário - 36571-000 - Viçosa, MG - nfsoares@ufv.br

${ }^{3}$ Engenheiro Agrônomo, Doutor, Professor - Escola de Agronomia e Engenharia de Alimentos/EAEA - Universidade Federal de Goiás/UFG - Rodovia Goiânia/Nova Veneza - Km 0 - Cx. P. 131 - 74001-970 - Goiânia, GO - robson.agro.ufg@gmail.com
} 
De acordo com Shorter (1982), se a taxa de oxidação do produto e a taxa de permeabilidade a $\mathrm{O}_{2}$ da embalagem forem conhecidas, torna-se possível calcular a quantidade de ferro requerida para manter o nível de $\mathrm{O}_{2}$ desejado durante o tempo de estocagem (LABUZA \& BREENE, 1989). Vários autores como Labuza (1987), Nakamura \& Hoshino (1983) e Vermeiren et al. (1999) relatam que 1g de ferro reagirá com $300 \mathrm{~cm}^{3}$ de $\mathrm{O}_{2}$.

Nakamura \& Hoshino (1983), com base na reação do ferro com o oxigênio, na pressão atmosférica sob diferentes umidades relativas, encontraram que, em geral, $1 \mathrm{~g}$ de ferro pode reagir com 0,0136 moles de oxigênio que equivale a um consumo de $3,36 \times 10^{-1} \mathrm{~L}$ de oxigênio, se o ferro estiver em solução e totalmente disponível.

Segundo Abbott (2002), as principais vantagens do uso dos absorvedores é a sua capacidade de reduzir os níveis de $\mathrm{O}_{2}$ para menos de $0,01 \%$, que é menor do que os tipicamente encontrados (0,3-3\%) nos sistemas tradicionais de atmosfera modificada, vácuo ou substituição da atmosfera interna por gás inerte. Assim, esse método tem despertado interesse como uma nova tecnologia de embalagem para preservar os alimentos, podendo substituir ou, principalmente, complementar as tecnologias empregadas para reduzir o nível de oxigênio no interior das embalagens (ABE \& KONDOH, 1989; NAKAMURA \& HOSHINO, 1983).

Para se obter maior efetividade dos absorvedores, algumas condições devem ser observadas, tais como, o uso de embalagens ou filmes com alta barreira a $\mathrm{O}_{2}$, apresentando taxa de permeabilidade a oxigênio de no máximo $20 \mathrm{~cm}^{3} \cdot \mathrm{m}^{-2} \cdot \mathrm{atm}^{-1} \cdot \mathrm{dia}^{-1}$. A selagem deve ser bem feita de modo que o $\mathrm{O}_{2}$ não se difunda através dela. Outro parâmetro importante é a seleção do absorvedor apropriado, tanto quanto ao tipo e ao tamanho (capacidade) a ser usado (NAKAMURA \& HOSHINO, 1983). Para essa seleção, o estado físico, a atividade de água do alimento, a quantidade de $\mathrm{O}_{2}$ dissolvido no produto e o nível inicial de $\mathrm{O}_{2}$ no espaço livre devem ser levados em consideração (SMITH et al., 1990, 1995).

Dessa forma, neste trabalho, objetivou-se determinar uma equação, para estimar o tempo de atividade do sachê, em função da taxa de permeabilidade a $\mathrm{O}_{2}$ da embalagem, umidade relativa e temperatura de estocagem do produto.

\section{MATERIAL E MÉTODOS}

Este trabalho foi realizado no Laboratório de Embalagens do Departamento de Tecnologia de Alimentos da Universidade Federal de Viçosa. O sistema absorvedor utilizado neste trabalho foi o absorvedor de oxigênio, à base de ferro, da marca O-Buster no formato de sachê. $\mathrm{O}$ modelo utilizado foi o FT-300, desenvolvido para ser utilizado em produtos com atividade de água (Aw) máxima de 0,85 , com capacidade, segundo o fabricante, de absorver até $300 \mathrm{~mL}$ de oxigênio. $\mathrm{O}$ experimento constituiu na determinação de uma equação para predizer o tempo teórico de atividade do sachê absorvedor de oxigênio, para a faixa de umidade relativa de $75 \%$ a $85 \%$ à temperatura ambiente $\left(25^{\circ} \mathrm{C} \pm 2{ }^{\circ} \mathrm{C}\right)$ e de refrigeração $\left(10^{\circ} \mathrm{C} \pm 2{ }^{\circ} \mathrm{C}\right)$. O tempo teórico de atividade do sachê é definido como o tempo em que o sachê apresentar a capacidade de absorver oxigênio maior que a taxa de permeabilidade a oxigênio da embalagem.

\section{Determinação da curva de absorção dos sistemas absorvedores em umidades relativas e temperaturas diferentes}

Em um sistema constituído de dissecadores, com capacidade de 5,5 litros, foi colocado um sachê absorvedor à base de ferro (FT-300, O-Buster). As tampas dos dissecadores continham septos de silicone por onde foram retiradas as amostras de ar, que eram homogeneizadas por meio de sistema de ventilação colocado dentro de cada dissecador. As amostras foram analisadas em um determinador de oxigênio da MOCON. Todo sistema foi mantido hermeticamente fechado.

Umidades relativas médias de 75,80 e $85 \%$ foram obtidas dentro dos dissecadores, utilizando-se soluções saturadas de cloreto de sódio, sulfato de amônio e cloreto de potássio, respectivamente. As amostras de ar foram coletadas, para o experimento conduzido a $25 \pm 2^{\circ} \mathrm{C}$, de $4 \mathrm{em}$ 4 horas, enquanto que, para o experimento sob temperatura de $10 \pm 2^{\circ} \mathrm{C}$, os intervalos foram de $12 \mathrm{em} 12$ horas. Em ambas as temperaturas, as amostras foram coletadas até se constatar que o sachê não apresentava mais capacidade de absorção de oxigênio. Dessa forma, foi então calculada a taxa de absorção dos sachês nas diferentes temperaturas, por meio de regressão linear. Assim, a partir do logaritmo da taxa de absorção, da umidade relativa e do volume absorvido foi gerada uma superficie de resposta da qual será possível predizer o tempo em que o absorvedor ainda apresenta atividade.

O experimento foi conduzido em modelo inteiramente casualizado com três repetições.

\section{RESULTADOS E DISCUSSÃO}

O conhecimento da taxa de absorção, associado ao valor da taxa de permeabilidade a $\mathrm{O}_{2}$ das embalagens 
plásticas, permite predizer o volume de oxigênio absorvido pelo sachê. Quando a taxa de absorção do sachê torna-se inferior à taxa de permeabilidade da embalagem, o teor de oxigênio dentro da embalagem começa a aumentar gradativamente. Assim, foi considerado, para a determinação das equações qual absorvedor apresentaria eficiência até que sua taxa de absorção fosse igual a taxa de permeabilidade da embalagem. Para se determinar o tempo teórico de atividade de absorção do sachê (Equações 5 e 6), foi considerado o volume de oxigênio absorvido pelo sistema (Tabela 1), a taxa de absorção do absorvedor e a umidade relativa, para as temperaturas de $25 \pm 2^{\circ} \mathrm{C}$ e $10 \pm 2^{\circ} \mathrm{C}$.

Para determinação do tempo teórico de absorção do oxigênio pelo sachê, dois fatores importantes devem ser levados em consideração, a área da embalagem (A) e o volume inicial ( $\mathrm{Vi}$ ) de oxigênio remanescente na embalagem após o envase, ou seja é o volume real ocupado pelo conteúdo ( $a r+$ produto), subtraindo então do volume do produto (dado pela densidade) multiplicado pelo teor de oxigênio no ar.

Os valores de Vi são determinados pela equação 3:

$$
V i=\pi\left(V t-\left(\frac{\mathrm{P}}{\mathrm{d}}\right)\right) * 0,204
$$

onde,

$\mathrm{Vt}=$ volume total do produto embalado, determinado por deslocamento de líquido $(\mathrm{mL})$

$\mathrm{P}=$ peso do produto embalado $(\mathrm{g})$

$\mathrm{d}=$ densidade do produto $(\mathrm{g} / \mathrm{mL})$

Assim, o tempo teórico estimado de atividade do, absorvedor, T, pode ser determinado pela equação 4 :

$$
T=\frac{V-V i}{T P_{02} * A}
$$

Eq. 4

Onde,

T: tempo teórico (dia)

$\mathrm{V}$ : volume em $\mathrm{mL}$, determinado pelas equações 1 e 2
Vi: volume inicial em $\mathrm{mL}$, determinado pela equação 3

$\mathrm{TP}_{\mathrm{O} 2}$ : permeabilidade da embalagem em $\mathrm{mL}$ de $\mathrm{O}_{2} \cdot \mathrm{m}^{-2} \cdot \mathrm{dia}^{-1}$ A: área da embalagem $\left(\mathrm{m}^{2}\right)$

Dessa forma, pode-se definir que, para a temperatura de $25 \pm 2^{\circ} \mathrm{C}$, o tempo teórico é definido pela seguinte equação:

$T=\frac{\left(-32,770+10,440 * \mathrm{UR}-104,385 * \ln \left(\mathrm{TPO}_{2}\right)\right)-\left[\mathrm{Vt}-\left(\frac{\mathrm{P}}{\mathrm{d}}\right)\right] * 0,204}{A^{*} T P O 2_{25^{\circ} \mathrm{C}}}$ Eq. 5

e para a temperatura de $10 \pm 2^{\circ} \mathrm{C}$

$T=\frac{\left(107,321+6,221 * \mathrm{UR}-105,166 * \ln \left(\mathrm{TPO}_{2}\right)\right)-\left[\mathrm{Vt}-\left(\frac{\mathrm{P}}{\mathrm{d}}\right)\right] * 0,204}{A * T P O 2_{10^{\circ} \mathrm{C}}}$ Eq. 6

Para exemplificar, consideremos duas embalagens, com composições diferentes, de 20x $25 \mathrm{~cm}$, com uma área de $0,05 \mathrm{~m}^{2}$ por face, uma delas com a permeabilidade de $8,63 \mathrm{~cm}^{3} \cdot \mathrm{m}^{-2} \cdot \mathrm{dia}^{-1}$ a $25{ }^{\circ} \mathrm{C}$ e a outra com a mesma permeabilidade a $10{ }^{\circ} \mathrm{C}$, o volume inicial de $\mathrm{O}_{2}$ no interior da embalagem, após envase do produto, de $2,5 \mathrm{~mL}$ e mantido em umidade relativa de $80 \%$ pode-se estimar o tempo teórico de atuação do sistema absorvedor para essas condições, usando as equações 5 e 6 . Assim, para a temperatura de $25 \pm 2^{\circ} \mathrm{C}$, o tempo estimado foi de 666 dias e para a de $10 \pm 2^{\circ} \mathrm{C}$ de 436 dias. Portanto, após esse tempo o absorvedor não apresentará mais efeito, pois a taxa de absorção será inferior à taxa de permeabilidade da embalagem.

A equação estimada para predizer o tempo de atividade dos absorvedores na faixa de 75 a $85 \%$ de umidade relativa e temperatura de $10 \pm 2^{\circ} \mathrm{C}$ e $25 \pm 2^{\circ} \mathrm{C}$ mostra a influência da taxa de permeabilidade $\mathrm{a}_{2}$ do filme utilizado no acondicionamento, na presença do absorvedor (Tabela 2).

Tabela 1 - Equações para determinação do volume máximo absorvido pelo sachê para as temperaturas de $10 \pm 2^{\circ} \mathrm{C}$ e $25 \pm 2^{\circ} \mathrm{C}$ e umidades relativas na faixa de 75 a $85 \%$.

\begin{tabular}{cccc}
\hline Temperatura & \multicolumn{1}{c}{ Equação } & $\mathrm{R}^{2}$ & $\mathrm{CC}^{*}$ \\
\hline $25^{\circ} \mathrm{C}$ (Eq. 1) & $\mathrm{V}=-32,770+10,440 * \mathrm{UR}-104,385 * \ln$ (Taxa) & 0,9151 & 0,9765 \\
$10^{\circ} \mathrm{C}$ (Eq. 2) & $\mathrm{V}=107,321+6,221 * \mathrm{UR}-105,166 * \ln$ (Taxa) & 0,8729 & 0,932 \\
\hline
\end{tabular}

Onde V: volume máximo de oxigênio absorvido em $\mathrm{mL}$.

UR: umidade relativa $(75-85 \%)$

Taxa : taxa de absorção do absorvedor ( $\mathrm{mL} \cdot$ dia $\left.^{-1}\right)$

* Coeficiente de correlação 
TABELA 2 - Influência da permeabilidade a $\mathrm{O}_{2}$ de filmes plásticos no tempo de absorção dos sachês, calculado pela equação 6.

\begin{tabular}{llcc}
\hline \multicolumn{1}{c}{ Filme } & \multicolumn{1}{c}{ Produto } & $\begin{array}{c}\text { Taxa } \\
\mathrm{mL} \cdot \mathrm{m}^{-2} \cdot \mathrm{dia}^{-1}\end{array}$ & Tempo (dia) \\
\hline PET/PE & Pré mistura para bolo & 110 & 28 \\
PET-PVDC/PE & Frutas e hortaliças desidratadas & 9 & 634 \\
BOPP/PE & Frutas e hortaliças desidratadas & 700 & 2 \\
PEBD & Minimamente Processado & 6500 & $<1$ \\
PVC & Minimamente Processado & 7000 & $<1$ \\
PP & Biscoito & 1700 & $<1$ \\
\hline
\end{tabular}

Fonte: Sarantópoulos et al. (2001), modificada.

Onde,

PET-politereftalato de etileno

$\mathrm{PE}$ - polietileno

PVDC - policloreto de vinilideno

BOPP - polipropileno biorientado

PVC - policloreto de vinila

$\mathrm{PP}$ - polipropileno

PEBD - polietileno de baixa densidade

\section{CONCLUSÃO}

Para predizer o tempo de absorção de oxigênio de sachês absorvedores de oxigênio utilizados em embalagens de alimentos, é necessário o conhecimento de alguns parâmetros, tais como: a taxa de permeabilidade a $\mathrm{O}_{2}$ da embalagem, a temperatura e a umidade relativa de estocagem do produto, bem como, o volume interno de oxigênio remanescente após envase do produto. Através da quantificação desses parâmetros foram calculadas duas equações para estimar o tempo de absorção de oxigênio de sachês absorvedores de oxigênio, quando usados em embalagens estocadas a 10 e $25{ }^{\circ} \mathrm{C}$ e na faixa de 75-85\% UR. As equações apresentaram coeficiente de ajuste elevado, podendo assim estimar o volume máximo absorvido pelo absorvedor de oxigênio usado no interior das embalagens de produtos alimentícios processados.

\section{AGRADECIMENTOS}

Os autores agradecem o apoio financeiro do $\mathrm{CNPq}$ e da FAPEMIG para a realização deste trabalho.

\section{REFERÊNCIAS BIBLIOGRÁFICAS}

$\mathrm{ABBOTT}, \mathrm{R}$. Intelligent paper packaging of unwrapped. Disponível em: 〈http://Www.pirapackaging.com》. Acesso em: 16 out. 2002.
ABE, Y.; KONDOH, Y. Oxygen absorbers. Trumbull: Food and Nutrition, 1989.

FLOROS, J. D.; DOCK, L. L.; HAN, J. H. Active packaging technologies and applications. Food Cosmetics and Drug Packaging, [S.1.], v. 20, p. 10-17, 1997.

LABUZA, T. P. Oxygen scavenger sachets. Food Research, Barking, v. 32, p. 276-277, 1987.

LABUZA, T. P.; BREENE, W. M. Applications of "active packaging" for improvement of shelf-life and nutritional quality of fresh and extended shelf-life foods. Journal of Food Processing and Preservation, [S.1.], v. 13, n. 1, p. 1-69, 1989.

MCKEDY, G. E. Oxygen absorber. Patente n. US 6,436,872 B2. Aug. 2002.

NAKAMURA, H.; HOSHINO, J. Technique for the preservation of food by employment of oxygen absorbers. Tokyo: Tokyo Ageless Division, 1983.

ROONEY, M. L. Active food packaging. Glasgow: Blackie Academic and Profession, 1995.

SARANTÓPOULOS, C. I. G. L.; OLIVEIRA, L. M.; CANAVESI, E. Requisitos de conservação de alimentos em embalagens flexíveis. [S.1.]: CETEA/ITAL, 2001. 213 p.

SHORTER, A. J. Evaluation of rapid methods for scavenging oxygen in flexible pouches. Lebensmittel Wissenschaft und Technologie, London, n. 15, p. 380-381, 1982.

SMITH, J. P.; HOSHINO, J.; ABE, Y. Interactive packaging involving sachet technology. In: ROONEY, M. L. Active food packaging. Glasgow: Blackie Academic and Profession, 1995. p. 143-173. 
SMITH, J. P.; RAMASWAMY, H. S.; SIMPSON, B. K. VERMEIREN, L.; DEVLIEGHERE, F.; BEEST, M. van; Development in food packaging technology, part 2: storage KRUIJF, N. de; DEBEVERE, J. Development in the active aspects. Trends of Food Science and Technology, packaging of foods. Trends in Food Science e Technology, Cambridge, v. 11, p. 111-118, 1990.

Cambridge, v. 10, p. 77-86, 1999. 